\title{
Evaluación del desarrollo de competencias a partir de la modelación matemática
}

\section{Assessment of the Development of Competences Based on Mathematical Modelling}

\author{
Carlos Márquez ${ }^{1}$, Carlos Gaviria ${ }^{2}$, Yohana \\ López $^{3}$
}

\author{
${ }^{1}$ Ciencias Básicas, Facultad de Ingenierías, Universidad de San Buenaventura, \\ Medellín, Colombia. Email: carlos.marquez@usbmed.edu.co \\ ${ }^{2}$ Ciencias Básicas, Facultad de Ingenierías, Universidad de San Buenaventura, \\ Medellín, Colombia. Email: carlos.gaviria@usbmed.edu.co \\ ${ }^{3}$ Ingeniería Industrial, Facultad de Ingenierías, Universidad de San Buenaventura, \\ Medellín, Colombia. Email: yohana.lopez@usbmed.edu.co
}

\section{OPEN ACCESS}

(9) $(\Theta \Theta$

Copyright:C) 2019 Ingenierías USBmed. La revista Ingenierías USBmed proporciona acceso abierto a todos sus contenidos bajo los términos de la licencia creative commons Atribución- no comercial- SinDerivar 4.0 Internacional (CC BY-NC-ND 4.0)

Tipo de Artículo: Investigación científica y tecnológica.

Recibido:24-05-2019.

Revisado: 14-06-2019.

Aprobado: 19-06-2019.

Doi: $\quad 10.21500 / 20275846.4132$

Referenciar así: C. Márquez, C. Gaviria \& Y. López. "Evaluación del desarrollo de competencias a partir de la modelación matemática". Ingenierías USBMed, 10(2), pp.8-15, 2019.

Declaración de disponibilidad de datos: Todos los datos relevantes están dentro del artículo, así como los archivos de soporte de información.

Conflicto de intereses: los autores han declarado que no existen conflicto de intereses.

Editores: Yohana López Rivera, Universidad de San Buenaventura, Medellín, Colombia. Alfonso Insuasti Rodríguez, Universidad de San Buenaventura, Medellín, Colombia. Erika Solange Imbett Vargas, Instituto Tecnológico Metropolitano. Eliana Zapata Ruiz, Instituto Tecnológico Metropolitano. José Fernando Valencia Grajales, Universidad Autónoma Latinoamericana.
Resumen. En el presente estudio se hizo una intervención didáctica en alumnos de grado once de la Institución Educativa San Vicente de Paúl de la ciudad de Medellín, con el objetivo de construir el concepto de función exponencial de manera significativa bajo el enfoque de situaciones problema. Para el estudio se utilizó un grupo experimental sobre el cual se realizó la intervención, y un grupo control con el que se trabajó de manera tradicional. A los alumnos se les suministró una secuencia de situaciones problema, en especial una de tipo experimental, mediante una unidad de enseñanza potencialmente significativa (UEPS) con diferentes grados de complejidad, para identificar los conocimientos previos e introducir los nuevos. En la práctica se propusieron situaciones de tipo gráfico, tabular y simbólico. Los resultados mostraron que los alumnos del grupo experimental alcanzaron mayores niveles de competencia que los que formaban parte del grupo control.

Palabras Clave. Aprendizaje significativo, situación problema, modelación, intervención didáctica, función exponencial, unidad de enseñanza potencialmente significativa.

Abstract. In the present study, an educational intervention was carried out in eleven graders belonging to the St. Vincent de Paul educational institution in the city of Medellín, in order to build the concept of exponential function in a meaningful way under the problem-situation approach. For the study, an experimental group, on which the intervention was carried out, was used, and a group control, which worked in the traditional way. Learners were supplied with a sequence of problem situations, especially one of the experimental type, through a Potentially Meaningful Teaching Unit (PMTU) with different degrees of complexity, in order to identify prior knowledge and to introduce the new one. In practice, graphical, tabular, and symbolic type situations were proposed. The results showed that learners in the experimental group reached higher levels of competence than those of the control group.

Keywords. Meaningful learning, problem situation, modeling, didactic intervention, exponential function, potentially meaningful teaching unit. 


\section{Introducción}

Durante muchas décadas se han realizado reformas en la educación que no han generado el impacto esperado en su calidad; generalmente, el papel del educador se reduce más a un "transmisor" que a un "mediador"; su práctica, basada en métodos de enseñanza tradicionales, encamina el proceso de enseñanza-aprendizaje hacia la transmisión-adquisición de conocimiento, con lo que acarrea un aprendizaje de tipo mecánico, dirigido más a la repetición y memorización de ideas que a la construcción de conceptos. Este enfoque tiene como modelo pedagógico el conductismo que, según Legorreta [1], es la modificación de la conducta a través de pares de estímulo-respuesta y refuerzo selectivo. En esta teoría no se consideran los procesos mentales y se mira el aprendizaje como la modificación de la conducta por la experiencia, y las consecuencias del reforzamiento y el castigo son parte de la vida del docente y los alumnos. Los docentes califican, elogian y reprenden; mientras que el alumno, desde esta perspectiva, se transforma en una persona que responde a los estímulos del medio ambiente, en tanto que la actividad que se le reconoce se refiere a la práctica, mental o física, que debe ejercer para lograr los aprendizajes [2]. Son múltiples los autores que coinciden que este tipo de práctica no es la adecuada cuando se pretende que un alumno aprenda de manera significativa y que desarrolle competencias cognitivas $[3,4,5,6]$.

Mejorar el proceso de enseñanza-aprendizaje es un reto para la educación actual: se debe comenzar a reestructurar y recontextualizar el currículo. Tal organización debe involucrar de manera explícita tres categorías: "qué enseñar", asunto relacionado con los inventarios de conocimientos; "cómo enseñar", que constituye un aspecto asociado con el dominio de estrategias didácticas; y "cuánto se enseña", lo cual indaga por las evidencias que arroja el proceso de aprendizaje. Estas categorías deben interactuar en permanente armonía [7] y articularse mediante una intervención didáctica bien estructurada, ya que todas deben aportar elementos para la adquisición de un verdadero aprendizaje significativo. En este sentido, Obando [4] sugiere que se debe organizar el currículo de tal manera que permita movilizar competencias a partir de una orientación metodológica participativa que integre otras alternativas diferentes a la presentación lineal y abstracta de los contenidos matemáticos.

La tendencia de la educación en la actualidad es la transición de un modelo educativo centrado en la enseñanza de datos hacia un modelo centrado en el aprendizaje significativo; eso significa que el protagonista en la construcción del conocimiento es el alumno [8]. En esta concepción el alumno pasa a ser el auténtico eje de la educación y el docente se constituye en organizador, guía y mediador en el encuentro del alumno con el conocimiento [9], es decir, solo debe generar un ambiente educativo enriquecido por la crítica y el sometimiento a la crítica [8] para que los alumnos por sí solos manifiesten una actividad cognitiva.

En consecuencia con lo anterior, uno de los grandes retos en la educación matemática es potenciar el pensamiento crítico y creativo de los aprendices. De acuerdo a Vizcarro y Juárez [10], un objetivo fundamental de la formación es que los estudiantes "aprendan a aprender" de forma independiente y sean capaces de adoptar de forma autónoma la actitud crítica que les permita orientarse en un mundo cambiante, lo cual hace necesario no solo cambiar la didáctica de la enseñanza sino en el tipo de aprendizaje que se desea construir. Se requieren intervenciones didácticas en el aula para mejorar los ambientes de aprendizaje que permitan al estudiante, por un lado, ser miembro activo en la construcción de su propio conocimiento; y por el otro, construir conceptos de manera significativa.

Una teoría que propicia elementos conceptuales para el aprendizaje del aula es la del aprendizaje significativo, la cual centra su atención en el estudiante y busca construir conceptos de manera significativa, aspecto de gran relevancia si se desean actuaciones exitosas en diversos contextos. Ausbel, citado en [5], plantea que el aprendizaje significativo es un proceso a través del cual una información se relaciona, de manera no arbitraria y sustantiva (no literal), con un aspecto relevante de la estructura cognitiva del individuo. En tal sentido, un aprendizaje es significativo cuando el aprendiz puede atribuir posibilidad de uso al nuevo contenido aprendido, relacionándolo con el conocimiento previo [11]. Es decir, los conocimientos previos sirven de "ancla" para los nuevos.

En esta perspectiva, la enseñanza requiere "metodologías activas" que propicien un espacio de interacción entre el estudiante, el objeto del conocimiento y el contexto. Tal interacción está mediada por el docente y tiene como fin el desarrollo de competencias. A propósito de estas últimas, en este trabajo una competencia se entiende tal como la define Bedoya [12, 13], esto es, como una actuación idónea que emerge en una tarea concreta, en un contexto con sentido. Esta actuación debe estar asociada con algún campo del saber; un estudiante es competente cuando pone en juego o en acción el saber. De una manera más explícita, la competencia es saber hacer en contexto con el conocimiento, es poner en acción el conocimiento aprendido; el fin de una competencia no es describir la realidad, sino modificarla. En este sentido, el ser competente debe dar respuesta al "saber que" y al "saber como", y en definitiva, la competencia se constituye en una propiedad del individuo en permanente modificación, condición que da el carácter dinámico al proceso de formación. Lograr esto requiere dar un giro hacia las propuestas de intervención en el 
aula. En el área de las matemáticas, las situaciones problema componen un contexto que permite dinamizar el aprendizaje porque dotan de significado a los conceptos matemáticos, con lo que permiten el aprendizaje del concepto y su aplicación. Las situaciones problema se pueden diseñar en diversos campos del saber: situaciones propias de las matemáticas y de otras áreas, en especial las ciencias naturales y de la vida, por ejemplo.

De lo anterior se deduce que alcanzar un desempeño exitoso en contexto a partir del conocimiento lleva consigo la condición de que el aprendizaje adquirido sea significativo; y este se logra cada vez que se consigue una interacción entre los conceptos previos y los nuevos conocimientos. David Ausubel [3] sugiere que para que se produzca aprendizaje significativo debe existir una interacción entre los conocimientos previos que el alumno tiene en su estructura cognitiva y los nuevos conocimientos. Tal interacción requiere que el material presentado al estudiante sea potencialmente significativo y despierte interés-una actitud para el aprendizaje--

Lo dicho hasta aquí supone que una intervención en el aula bajo el enfoque de situaciones problema permite movilizar competencias matemáticas, en busca de potenciar el pensamiento matemático a través de procesos de conceptualización y simbolización [14]. En este sentido, plantea Rúa [15], que una situación problema es un espacio de interrogantes frente a los cuales el sujeto está convocado a responder. En el campo de las matemáticas, una situación problema se interpreta como un espacio pedagógico que posibilita la conceptualización, la simbolización y la aplicación comprensiva de algoritmos en aras de plantear y resolver problemas.

La situación problema es el detonador de la actividad cognitiva; para que esto suceda debe involucrar de forma implícita los conceptos que se van a aprender y representar un verdadero "problema" para el estudiante, pero a la vez debe ser accesible y permitir que se utilicen conocimientos anteriores [16].

Perrenoud, citado por [17], concibe la competencia íntimamente ligada a una situación o familia de situaciones: la dupla competencia y situación es disociable, por cuanto la primera está siempre referida a la segunda o a una familia de ellas.

En consonancia con lo expuesto, se realizó en el marco del presente trabajo una intervención de aula constituida por un conjunto de situaciones problema, cuyo propósito es evaluar el desarrollo de competencias cognitivas a partir de la construcción de aprendizajes significativos. Las competencias que se desarrollaron son la resolución y planteamiento de problemas, razonamiento, comunicación, modelación, elaboración, comparación y ejercitación de procedimientos.

Habida cuenta de que la función exponencial es un modelo que permite representar la dinámica de diversos sistemas complejos y que es un concepto de difícil aprehensión, resulta importante evaluar la utilidad de propuestas pedagógicas orientadas a la solución de situaciones problema que pongan en acción el concepto de la función exponencial para alcanzar altos niveles de competencia en los estudiantes.

Por esta razón, el objetivo de este trabajo fue evaluar el impacto de la modelación exponencial en situaciones problema de tipo experimental en la adquisición de aprendizaje significativo en estudiantes de grado 11.

\section{Materiales y métodos}

Se realizó un estudio bajo enfoques cuantitativo y cualitativo. Respecto del primero se condujo un estudio descriptivo en el que se utilizaron inferencias paramétricas para el análisis de resultados; mientras que en el caso del segundo se utilizaron tablas de contingencia para el análisis de la independencia de las variables.

\section{A. Participantes}

La población objeto de estudio correspondió a los alumnos de grado 11 de la institución educativa San Vicente de Paúl. El tamaño de la población ascendió a 252 alumnos, de los cuales se extrajeron dos muestras aleatorias para conformar un grupo control de tamaño $n=65$ y un grupo experimental de tamaño $n=62$. Las muestras se seleccionaron mediante muestreo aleatorio simple. Para la selección de los integrantes de cada muestra se generaron números aleatorios en el software $R^{\circledR}$. La variable más relevante en el estudio es la medición del nivel de competencia alcanzado por los estudiantes luego que el grupo experimental fuera sometido a un intervención didáctica, cuyo fin fue la construcción de manera significativa del concepto de función exponencial. Para realizar la intervención didáctica se diseñó una unidad de enseñanza potencialmente significativa (UEPS) [5], que contenía un conjunto secuencial de situaciones problema a través de las cuales se pusieron en interacción los conocimientos previos y nuevos - que se pretendían que el estudiante adquiriera-. Tales situaciones problema se presentaron de menor a mayor complejidad; la de más relevancia fue una de carácter experimental [18], toda vez que se buscaba un mejor contexto para el aprendizaje. Los alumnos trabajaron en grupos de cuatro personas y luego se realizó una socialización dirigida por el docente.

\section{B. Herramientas o materiales}

En el desarrollo de la intervención didáctica se utilizaron la plataforma Moodle para la administración de los contenidos temáticos y las situaciones propuestas, 10 tarjetas de adquisición de datos Arduino, los programas Statgraphics 5.1 Plus ${ }^{\circledR}$, Epi Info $7^{\circledR}$ y Excel ${ }^{\circledR}$, protoboards, condensadores fijos, variables electrolíticos, resistencias de 10, 100 y $1000 \Omega$, baterías de 9 voltios, computadores personales y cables. 


\section{Procedimiento}

El trabajo se orientó en tres etapas:

a) Etapa preactiva:

Esta correspondió a la planeación y diseño de las situaciones problema por parte del docente, basado en las competencias que propone el microcurrículo de la asignatura y las evaluaciones que darían cuenta del nivel de competencia alcanzado.

b) Etapa activa

Durante esta etapa de encuentro con los estudiantes se desarrolló la intervención de aula, en tres fases:

1. Inicio: tuvo como objetivo retraer los conceptos previos que los alumnos tenían en la estructura cognitiva con respecto al concepto de función. Por esta razón, se propusieron situaciones problema sobre las funciones lineal, cuadrática y exponencial, que permitieron evidenciar el estado inicial de los estudiantes en cuanto a sus pre conceptos y competencias básicas. Para el estado encontrado fue necesario construir un organizador previo sobre los temas propuestos; en esta fase, el docente repasa los conceptos y los alumnos los recuerdan; luego, se les aplica una prueba para evidenciar su avance y finalmente se les suministra dos situaciones problema de cada tema en su orden, función lineal, función cuadrática y función exponencial, que fueron evaluadas al terminar cada tema.

2. Desarrollo: en esta fase se buscó poner en interacción los conocimientos nuevos y previos. Se desarrollaron situaciones problema de tipo experimental, con las cuales se modeló un fenómeno de respuesta exponencial. En la misma línea, se realizó una comparación entre los resultados experimentales y aquellos arrojados por la simulación computacional a través de los modelos matemáticos que representan la conducta de los fenómenos estudiados.

3. Terminación: se recopilaron las evidencias del aprendizaje de los estudiantes, a través de tests que contenían situaciones problema; posteriormente, se analizaron los resultados y se produjeron los informes del nivel de competencia alcanzado.

c) Etapa posactiva

Se evalúan aciertos y desaciertos en la metodología utilizada y se elaboran recomendaciones.

\section{d) Descripción de los tests}

Con el objetivo de acopiar evidencia del aprendizaje de los estudiantes, se aplicó un test de ocho preguntas a los grupos experimental y de control, a fin de evaluar los conceptos que se proponía construir de manera significativa.

La información generada por el test proporciona dos puntos de vista para el análisis:
1. Cualitativo: según las respuestas de cada estudiante al test se clasificaron en tres niveles, de menor a mayor grado de complejidad. Estos son inclusivos, es decir, los estudiantes que están en el nivel 2 también han superado el 1 y aquellos que están en el 3 también han superado los dos primeros.

2. Cuantitativo: a partir de las respuestas del mismo test se obtuvo una calificación de 0 a 5 , con la cual se pretende comparar la nota promedio entre los grupos experimental y control.

\section{Análisis estadístico}

Para el análisis estadístico se utilizaron los software Statgraphics 5.1 Plus@ y Epi Info 7@.

El tratamiento de la información se realizó mediante dos tipos de análisis:

a) Análisis univariado

Los niveles de competencia se expresaron en porcentajes en tablas de frecuencia, posteriormente graficadas.

b) Análisis bivariado

Se utilizó la prueba de Kolmogorov-Smirnov para probar que los datos provienen de una distribución normal, y la prueba de independencia Chi-cuadrado [19] para determinar la asociación entre el nivel de competencia y el tipo de intervención. Se calcularon los RR con intervalo de confianza del $95 \%$ y se consideró significativo un valor de $p<0.05$. Adicionalmente, se realizó una comparación entre las medias de las notas de $0-5$ entre ambos grupos a través de una prueba $t$ de Student.

\section{Resultados}

A. Niveles de competencia de los grupos control y experimental

Las tablas 1 y 2 muestran los niveles de competencia exhibidos por dos grupos después de aplicar el test de conocimiento. La primera columna muestra la frecuencia absoluta de individuos según el nivel máximo de competencia logrado; y la segunda, la frecuencia acumulada "mayor o igual que", en virtud del mencionado carácter inclusivo de los niveles. La última columna, muestra el porcentaje para esa frecuencia acumulada.

Tabla 1. Niveles de competencia para el grupo experimental

\begin{tabular}{|c|c|c|c|}
\hline \multicolumn{4}{|c|}{ Superación de niveles del grupo control } \\
\hline \hline Competencia & Frec. absoluta & Frec. acumulada & $\%$ \\
\hline Nivel 1 & 19 & 65 & $100 \%$ \\
\hline Nivel 2 & 33 & 46 & $71 \%$ \\
\hline Nivel 3 & 13 & 13 & $20 \%$ \\
\hline Total & 65 & 124 & \\
\hline
\end{tabular}

En la figura 1 se muestra la superación de los niveles de competencia en ambos grupos. En ella se puede apreciar que en el nivel 1 el grupo experimental estuvo muy cerca del valor esperado, mientras que el grupo 
Tabla 2. Niveles de competencia para el grupo experimental Superación de nivel grupo experimental

\begin{tabular}{|c|c|c|c|}
\hline \multicolumn{4}{|c|}{ Superación de nivel grupo experimental } \\
\hline \hline Competencia & Frec. absoluta & Frec. acumulada & $\%$ \\
\hline Nivel 1 & 3 & 62 & $95 \%$ \\
\hline Nivel 2 & 18 & 59 & $91 \%$ \\
\hline Nivel 3 & 41 & 41 & $63 \%$ \\
\hline Total & 62 & 162 & \\
\hline
\end{tabular}

control alcanzó el 100\%. Esto podría estar asociado con la tendencia que presentó el grupo experimental a trabajar de forma preferencial las preguntas relacionadas con los niveles 2 y 3 . Este hecho es muy significativo, ya que el grado de complejidad de las preguntas en estos niveles es mayor; y podría sugerir que la intervención didáctica causa más impacto en estos niveles porque hubo mayor adquisición del aprendizaje.

\section{SUPERACIONDE NIVELES}

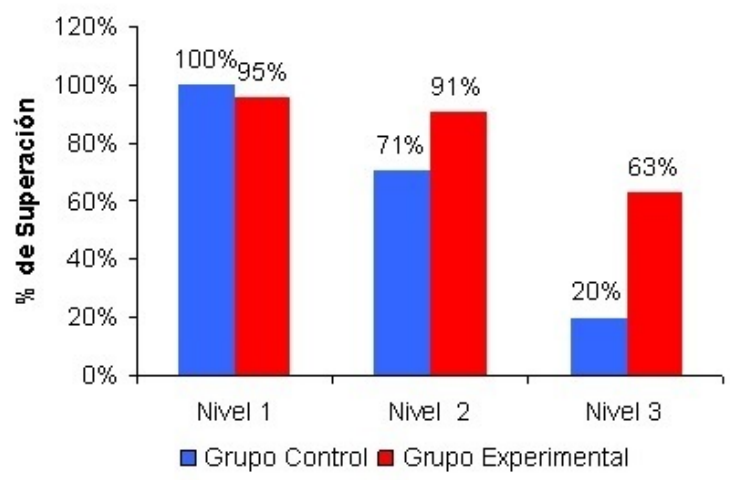

Figura 1. Comparación entre los niveles de competencia de los grupos control experimental

B. Comparación entre niveles de competencia, según el grupo

Este análisis se realizó mediante tablas de contingencia, con el fin de analizar la relación de dependencia o independencia entre los 3 niveles de competencia, así como el tipo de intervención realizada. Se consideran las siguientes hipótesis:

$H_{0}$ : El nivel de competencia alcanzado es independiente del grupo en el que se encuentre el estudiante.

$H_{a}$ : El nivel de competencia alcanzado es dependiente del grupo en el que se encuentre el estudiante.

Tabla 3. Niveles de competencia, según el grupo

\begin{tabular}{|c|c|c|c|c|}
\hline Grupo & N1 & N2 & N3 & Total \\
\hline Experimental & 62 & 59 & 41 & 162 \\
\hline Control & 65 & 46 & 13 & 124 \\
\hline Total & 127 & 105 & 54 & 286 \\
\hline
\end{tabular}

Para buscar asociación entre los niveles de competencia según el grupo, se aplicó una prueba Chi-cuadrado con 2 grados de libertad a los datos consignados en la tabla 3. Debido a que el valor calculado 11.35 es mayor que el valor crítico 5.99, se rechaza $H_{0}$ y se concluye que hay dependencia entre el nivel de competencia y el grupo al cual pertenece el alumno.

Para buscar la fuerza de la asociación entre pertenecer a un grupo y el nivel de competencia, se calculó el riesgo relativo $(R R)$ con su respectivo intervalo de confianza $(I C)$ a través de tablas de contingencia como la mostrada en la tabla 4 a través del software Epi Info $7^{\circledR}$ y la ecuación (1).

Tabla 4. Cálculo de RR

\begin{tabular}{|c|c|c|c|}
\hline \multicolumn{4}{|c|}{ Nivel de competencia } \\
\hline \multirow{3}{*}{ Grupo } & & N1 & N2 \\
\hline & Experimental & $a$ & $b$ \\
\hline & Control & $c$ & $d$ \\
\hline & $\ell=\frac{\frac{a}{a+b}}{\frac{c}{c+d}}$ & (1) & \\
\hline
\end{tabular}

C. Nivel de competencia N1 vs. N2, según grupo

Tabla 5. Tabla de contingencia N1 vs. N2

\begin{tabular}{|c|c|c|c|}
\hline \multicolumn{4}{|c|}{ Nivel de competencia } \\
\hline \multirow{3}{*}{ Grupo } & & N1 & N2 \\
\cline { 2 - 4 } & Experimental & 62 & 59 \\
\cline { 2 - 4 } & Control & 65 & 46 \\
\hline
\end{tabular}

De la tabla 5 se obtuvieron $R R=0.8750, p=0.1336$ e $I C(0.6925,1.1056)$, con un nivel de confianza $95 \%$. Dado que el intervalo de confianza del $R R$ incluye el 1 y el valor de $p$ es superior a 0.05 , se concluye, con un nivel de confianza del 95\%, que no hay asociación entre el nivel de competencia 1 y el hecho de pertenecer al grupo experimental.

D. Nivel de competencia N3 vs. N2 según, grupo

Tabla 6. Tabla de contingencia N3 vs. N2

\begin{tabular}{|c|c|c|c|}
\hline \multicolumn{4}{|c|}{ Nivel de competencia } \\
\hline \multirow{3}{*}{ Grupo } & & N3 & N2 \\
\cline { 2 - 4 } & Experimental & 41 & 59 \\
\cline { 2 - 4 } & Control & 13 & 46 \\
\hline
\end{tabular}

De la tabla 6 se obtuvieron $R R=1.8608, p=0.0108 \mathrm{e}$ $I C(1.0904,3.1755)$ con un nivel de confianza 95\%. Dado que el intervalo de confianza del $R R$ no contiene el 1 y el valor de $p$ es superior a 0.05 se encuentra significado estadístico: un RR cercano a 2 indica que por cada alumno del grupo control que pasa al nivel 3, 2 del experimental pasan al nivel 3 con un nivel de confianza del $95 \%$.

E. Nivel de competencia N3 vs. N1, según grupo

Tabla 7. Tabla de contingencia N3 vs. N2

\begin{tabular}{|c|c|c|c|}
\hline \multicolumn{4}{|c|}{ Nivel de competencia } \\
\hline \multirow{3}{*}{ Grupo } & & N3 & N1 \\
\cline { 2 - 4 } & Experimental & 41 & 59 \\
\cline { 2 - 4 } & Control & 13 & 46 \\
\hline
\end{tabular}

De la tabla 7 se obtuvieron los resultados $R R=2.3883$, $p=0.00054$ y $I C(1.3778,4.1402)$ con un nivel de confianza $95 \%$. Dado que el intervalo de confianza del $R R$ 
contiene el $1 \mathrm{y}$ el valor de $p$ es menor de 0.05 se encuentra significado estadístico: como en el caso anterior, un $R R$ cercano a 2 , indica que por cada alumno del grupo control que pasa al nivel 3, 2 del experimental lo logran con un nivel de confianza del $95 \%$.

F. Comparaciones de medias de las notas de ambos grupos

Con el fin de comparar el desempeño promedio en cuanto a nivel de competencia alcanzado entre los grupos control y experimental, se utilizó la prueba $t$ de Student. Antes de ello, se demostraron los siguientes supuestos:

1. Escogencia aleatoria: la escogencia de ambos grupos se hizo de forma aleatoria a través de muestreo aleatorio simple. De cuatro grupos de grado 11 se escogieron dos para el grupo experimental y dos para el grupo control.

2. Prueba de normalidad: se aplicó la prueba de KolmogorovSmirnov para demostrar que los datos provienen de una distribución normal.

3. Prueba de homocedasticidad: es decir, homogeneidad de la varianza del nivel de desempaño en los dos grupos, mediante la prueba de Levene.

G. Prueba de normalidad

a) Grupo control

Se consideran las siguientes hipótesis:

$H_{0}$ : Los datos del grupo control no tienen una distribución normal.

$H_{a}$ : Los datos del grupo control tienen una distribución normal.

Tabla 8. Prueba de normalidad para el grupo control, usando Statgraphics 5.1 Plus ${ }^{\circledR}$

\begin{tabular}{cccc}
\hline EDF Statistic & Value & Modified Form & P-Value \\
\hline S-K & 0.280358 & 2.28707 & $<0.01 *$ \\
Anderson-Dar $A^{2}$ & 3.66902 & 3.71331 & $0.0000 *$ \\
\hline
\end{tabular}

Según la prueba de Kolmogorov-Smirnov [20], en la tabla 8 , la md (máxima diferencia) es de 0.280358 y es mayor que el valor crítico de $p=0.01$; por lo tanto, se rechaza $H_{0}$ y se concluye que los datos se distribuyen normalmente, con un nivel de confianza del $99 \%$.

b) Grupo experimental

Se consideran las siguientes hipótesis:

$H_{0}$ : los datos del grupo experimental no tienen una distribución normal.

$H_{a}$ : los datos del grupo experimental tienen una distribución normal.

Tabla 9. Prueba de normalidad para el grupo control, usando Statgraphics 5.1 Plus ${ }^{\circledR}$

\begin{tabular}{cccc}
\hline EDF Statistic & Value & Modified Form & P-Value \\
\hline S-K & 0.172145 & 1.37233 & $<0.01 *$ \\
Anderson-Dar $A^{2}$ & 2.32564 & 2.35513 & $0.0000 *$ \\
\hline
\end{tabular}

Según la prueba de Kolmogorov-Smirnov, en la tabla
9 la md es de 0.172145 , y es mayor que el valor crítico de $p=0.01$; por tanto, se rechaza $H_{0}$ y se concluye que los datos se distribuyen normalmente, con un nivel de confianza del $99 \%$.

H. Prueba de homocedasticidad

La comparación de las varianzas de las dos muestras por la prueba de Levene [21] obtuvo los siguientes valores:

Ratio of Variances: [0.509887,1.3875].

$F=0.842392 P$-value $=0.498323$.

Dado que el intervalo de confianza calculado con un nivel de confianza del $95 \%$ se extiende desde 0.509887 hasta 1.3875 para la relación de las varianzas y que contiene el 1 , no existe una diferencia significativa entre estas últimas.

I. Prueba $t$ de Student para comparación de medias

Se consideran las siguientes hipótesis:

$H_{0}$ : Las medias de los dos grupos son iguales.

$H_{a}$ : Las medias de los dos grupos son diferentes.

Tabla 10. Prueba t de student, resultados de Statgraphics

\begin{tabular}{lcc}
\multicolumn{3}{c}{5.1 Plus ${ }^{\circledR}$} \\
\hline & Control & Experimental \\
\hline Count & 65 & 62 \\
Average & 2.97538 & 3.74839 \\
Variance & 0.7790728 & 0.924833 \\
Stand desv & 0.882651 & 0.961683 \\
95\% CI mean & {$[2.75667,3.1941]$} & {$[3.50416,3.99261]$} \\
\hline
\end{tabular}

Según los resultados de la tabla 10, existe una diferencia estadística en las medias de ambos grupos. La comparación de los intervalos de confianza indican que el desempeño del grupo experimental comprendido entre 3.50416 y 3.99261 fue significativamente mayor que el desempeño en el grupo control en el que el intervalo está comprendido entre 2.75667 a 3.1941 .

El análisis de comparación de medias por medio de la prueba $t$ de Student [22] obtuvo los valores de: $t=-4.72248$ y $P$-value $=0.00000631785$, consideradas varianzas iguales y habida cuenta de las siguientes hipótesis:

$$
H_{0}: \text { media } 1=\text { media } 2 \text { vs } H_{a}: \text { media } 1 \neq \text { media } 2
$$

Dado que el valor $p$ es inferior a 0.05 , se rechaza $H_{0}$ y se concluye que las medias de los dos grupos son diferentes, con una confianza del $95 \%$.

\section{Discusión}

A partir de los hallazgos encontrados, aceptamos la hipótesis alternativa: el desempeño de los estudiantes sometidos a intervención didáctica mejora significativamente con respecto al desempeño de los estudiantes a través del método tradicional. Los resultados muestran que fue posible lograr un impacto en el aprendizaje significativo de los estudiantes que fueron sometidos a dicha intervención. 
Estos resultados guardan relación con lo que sostienen Rodríguez [23], Caballero [24], Guerra [25] y Munera [26], quienes señalan que la forma como se produce el aprendizaje significativo tiene que ver con la manera en que se presenten los conceptos que se pretende que los alumnos aprendan. Estos autores expresan que para aprender conceptos de manera significativa se debe crear un entorno apropiado para lograr la interacción entre los conceptos previos y los nuevos conocimientos, reflexión acorde con lo que se ha encontrado en este estudio.

El uso de fenómenos con respuesta exponencial como contexto para construir el concepto de función exponencial de manera significativa fue un acierto ya que permitió simular una comunidad de aprendizaje, en la que se conjugaron todos los actores que deben intervenir en el acto educativo propuesto por la teoría del aprendizaje significativo. Igualmente, constituye en una propuesta innovadora que debería ser tenida en cuenta en el futuro.

Lo que no concuerda con los trabajos realizados de los autores referidos, bajo el enfoque de situaciones problema, es que con frecuencia presentan orientación más hacia el contexto matemático pero no proporcionan la experimentación directa como un espacio para la construcción de aprendizaje significativo, como articulación de otras áreas de las ciencias.

Esta experiencia didáctica sugiere que se puede lograr aprendizaje significativo con muy buenos resultados en la enseñanza de las Matemáticas mediante la construcción de situaciones problema de tipo experimental en el campo de las Ciencias Naturales, ya que existe multiplicidad de fenómenos que pueden servir como contexto para construir conceptos matemáticos.

\section{Conclusiones y Recomendaciones}

\section{A. Conclusiones}

Los resultados de este trabajo ponen de manifiesto un campo de acción interesante para futuras investigaciones, las cuales pueden enfocarse para resolver preguntas tales como: ¿es posible mejorar la enseñanza-aprendizaje a partir de situaciones problemas de tipo experimental? ¿Una intervención didáctica de este tipo mejora nivel de competencia de los estudiantes? ¿Hay mejoramiento en el ambiente de aprendizaje de los estudiantes a través de este tipo de intervención?

\section{B. Recomendaciones}

La investigación encuentra en la Institución Educativa San Vicente de Paúl unos puntos álgidos en el área de Matemáticas, por lo que se recomienda:

1. Reestructurar el currículo de matemáticas en la Institución y darle una orientación hacia el desarrollo de situaciones problemas con énfasis en la experimentación directa y su constatación con la simulación de modelos matemáticos.

2. Capacitar a los docentes del área en esta metodología para mejorar los niveles de desempeño de los estudiantes tanto en pruebas internas como en las externas.

3. Trabajar con este tipo de intervención desde los primeros años de enseñanza, para que los alumnos adquieran una cultura de aprendizaje a través de situaciones problemas.

4. Facilitar los instrumentos y los espacios diferentes al aula de clases para llevar a cabo los laboratorios.

\section{Referencias}

[1] B. Legorreta, "Enfoques y fundamentos de las teorías de aprendizaje.," 1993.

[2] P. Gómez, "Teoría del aprendizaje y formación inicial de profesores.," 1993.

[3] D. Ausubel, Psicologia educativa. Un punto de vista cognitivo. México: Trillas., 1976.

[4] G. Obando, "Las situaciones problemas como estrategías para la conceptualización matemática," Eduación y pedagogía, vol. XV, no. 35, p. 18, 2003.

[5] M. Moreira, Teoría del aprendizaje Significativo. Porto Alegre: Editora da Universida de Porto Alegre, 1993.

[6] J. E. Castrillón Parra, M. J. Camacho Amariles, and C. A. Castro Castro, "Aprendizaje basado en problemas en el camino a la innovación en ingeniería," Revista Ingenierías USBMed, vol. 7, no. 2, pp. 96-103, 2016.

[7] P. Ahumada, La evaluación en la concepción del aprendizaje significativo. (1. ${ }^{a}$ ed.). Valparaíso, Chile: Universidad de Valparaíso, 2001.

[8] E. Zuleta, Lógica y critica. Bogotá D.C.: Hombre nuevo editores, 2010.

[9] F. Díaz, Estrategias docentes para un parendizaje significativo:Una interpretación constructivista. 2002.

[10] C. Vizcarro and E. Juárez., La metodología del aprendizaje basado en problemas. Universidad Autónoma de Madrid, 2006.

[11] J. Rivera, "El aprendizaje significativo y la evaluación de los aprendizajes," Revista investigación eduacativaí, vol. 8, pp. 48-52, 2004.

[12] M. D. Bedoya, "Competencias y proyecto pedagógico," 2000.

[13] G. Gómez, L. Fernanda, and L. Yohana, "Propuesta lúdica como herramienta de apoyo al proceso enseñanza-aprendizaje en el estudio del trabajo, enfocada a la estandarización de tiempos," Ingenierías USBMed, vol. 9, no. 2, pp. 34-43, 2018.

[14] B. Mesa, Contextos para el diseño de situaciones problemas en la enseñanza de las matemáticas. Medellín: U de A, 1998.

[15] J. Rúa, "Un modelo de situación problema para la 
evaluación de competencias matemáticas," Entre Ciencia e Ingeniería, no. 4, pp. 9-37, 2008.

[16] L. Moreno, Fundamentación Cognitiva del currículo de Matemáticas. México: Seminario nacional de formación docente: Uso de nuevas tecnologías, 2002.

[17] J. Vaca and V. Aguilar, ¿Qué demonios son las competencias? Aportaciones del constructivismo clásico y contemporáneo. México: Universidad Veracruzana, 2015.

[18] J. Perales, "La resolución de problemas en la didáctica de las ciencias experimentales," Revista de educación y pedagogía, vol. 21, pp. 119-144, 1998.

[19] M. Gómez, "Cómo seleccionar una prueba estadística," Revista Méxicana de Pediatría, vol. 80, pp. 8283, 2013.

[20] I. Pedrosa, "Pruebas de bondad de ajuste en distribuciones simítricas, ¿qué estadístico utilizar," Universitas psychologica, vol. 14, p. 247, 2015.

[21] J. Correa, "Estudio de potencia de pruebas de homogeneidad de varianza," Revista Colombiana de Estadística, vol. 29, pp. 57-76, 2006.

[22] W. Mendenhall, Probabilidad y Estadística para Ingeniería y Ciencias. (Cuarta ed.). México: Prentice Hall, 1997.

[23] M. Rodríguez, La teroría del aprendizaje significativo. 2008.

[24] M. Cabalero, La progresividad del aprendizaje significativo de conceptos. 2008.

[25] F. Guerra, "Las situaciones problemas mediadoras de aprendizajes significativos de la función lineal," 2013.

[26] J. Múnera, "Las situaciones problema como fuente de matemátización.," Cuadernos pedagogícos, vol. 16, pp. 48-52, 2001. 